\title{
Blocking the drivers
}

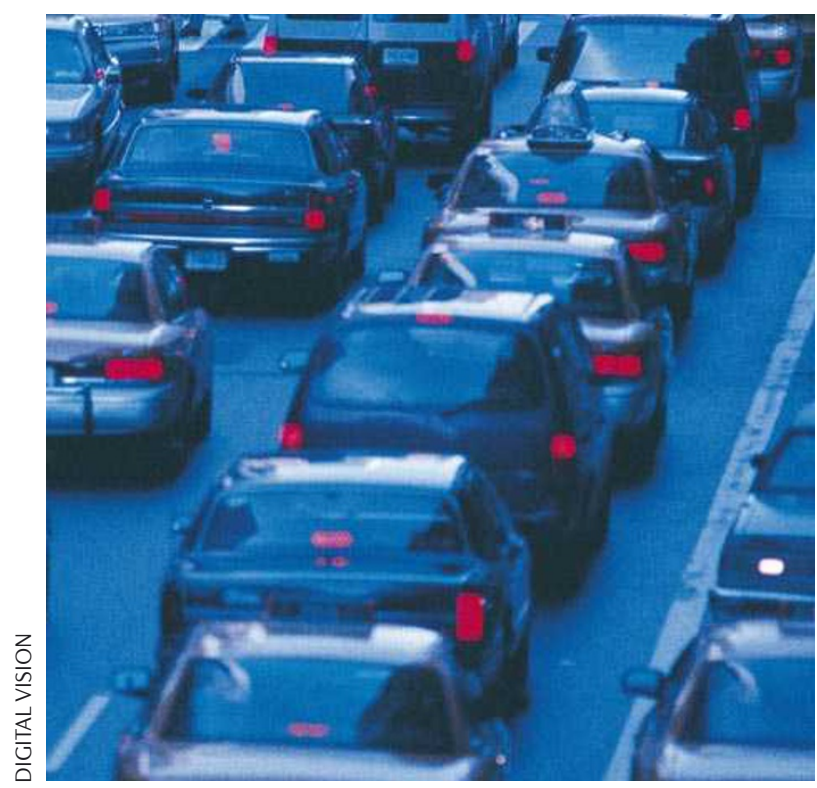

Psoriasis - a chronic autoimmune skin disease characterized by heavily scaled red plaques - is currently incurable. Moreover, available therapies are associated with issues such as inconvenience, limited efficacy, toxicity and high cost. Now, writing in the Journal of Clinical Investigation, Skvara and colleagues provide evidence indicating that a protein kinase $\mathrm{C}$ ( $\mathrm{PKC})$ inhibitor, AEB071, has potential as a novel treatment for this condition that might help address some of the drawbacks with current drugs.

Although the exact cause of psoriasis remains incompletely understood, it is thought to be predominantly driven by the infiltration of $\mathrm{T}$ cells into the skin, with more recent data implicating a contributory pathogenic role for resident skin cells such as keratinocytes. The PKC family have a central role in cell signalling, participating in proliferation, differentiation, migration, survival and death. Notably, specific $\mathrm{PKC}$ isoforms regulate such processes in these psoriasis-associated cells. This knowledge led the authors to propose that AEB071, being a potent inhibitor of various classic and novel $\mathrm{PKC}$ isoforms, may inhibit the drivers of psoriasis pathogenesis.

Initial in vitro studies examining the activity of AEB071 on cells involved in autoimmune diseases revealed that AEB071 inhibits T-cell proliferation and abolishes cytokine production from activated human T cells, macrophages and keratinocytes. Specifically, interleukin 17 (IL17) - known to be highly upregulated in psoriasis skin and a pro-inflammatory cytokine for keratinocytes - was potently inhibited. In addition, AEB071 proved to be efficacious in an in vivo model of skin inflammation, preventing an acute allergic T-cell driven contact dermatitis reaction in rats, which further strengthened their hypothesis.

Next, Skvara and colleagues went on to test whether their theory translated into the clinic. First, in 48 healthy human volunteers, a single oral dose of AEB071 (up to 500 $\mathrm{mg}$ ) was shown to be well tolerated, exerting a dose-dependent decrease in T-cell proliferation and IL2 mRNA expression in peripheral blood. The authors therefore predicted that AEB071 might be effective in a human disease that is associated with T-cell activation, such as psoriasis.

Indeed, a subsequent study in 32 patients with moderate to severe psoriasis, similarly revealed that AEB071 is well tolerated. AEB071 exerted a dramatic improvement in psoriasis symptoms after 2 weeks of twice-daily treatment (measured by a $69 \%$ mean reduction in psoriasis area severity index scoring). Skin was almost normal under histological examination, exhibiting a marked reduction of plaque infiltration of T cells and of proliferating keratinocytes. Furthermore, epidermal thickening and the number of $\mathrm{p} 40^{+}$dermal cells, which are also known to have a role in the disease, were decreased.

In addition to its promise as a novel psoriasis therapy, the capacity of AEB071 to differentially inhibit a range of $\mathrm{PKC}$ isoforms and act on various immune cell types suggests that it has potential therapeutic applications in a range of autoimmune conditions.

Sarah Crunkhorn

ORIGINAL RESEARCH PAPER Skvara H. et al. The PKC inhibitor AEB071 may be a therapeutic option for psoriasis.J. Clin. Invest. 7 Aug 2008 (doi:10.1172/JCI35636) 